\title{
Borne sur le degré des polynômes presque parfaitement non-linéaires
}

\author{
François Rodier*
}

\begin{abstract}
Résumé
Nyberg a défini la notion de la non-linéarité presque parfaite (APN) pour caractériser les fonctions qui ont la meilleure résistance aux attaques différentielles. Nous montrons ici que pour beaucoup de fonctions booléennes APN, le nombre de ses variables est borné par une expression dépendant du degré de cette fonction.

\section{English extended abstract}

The vectorial Boolean functions are employed in cryptography to build block coding algorithms. An important criterion on these functions is their resistance to the differential cryptanalysis. Nyberg defined the notion of almost perfect non-linearity (APN) to study resistance to the differential attacks. Up to now, the study of functions APN was especially devoted to the function powers. Recently some people showed that certain quadratic polynomials were APN.

Here, we will give a criterion so that a function is not almost perfectly non-linear.

H. Janwa showed, by using Weil's bound, that certain cyclic codes could not correct two errors. A. Canteaut showed by using the same method that the functions powers were not APN for a too large value of the exponent. We use Lang and Weil's bound and a result of P. Deligne (or more exactly an improvement given by Ghorpade and Lachaud) on the Weil's conjectures about surfaces on finite fields to generalize this result to many polynomials. We show therefore that these polynomials cannot be APN if their degrees are too large. We study many examples, and make some computation, showing that one does not get any new APN function for a polynomial on a field with at most 512 elements.
\end{abstract}

*Institut de Mathématiques de Luminy - C.N.R.S. - e-mail : rodier@iml . univ-mrs .fr 


\section{Introduction}

Les fonctions booléennes vectorielles sont employées dans la cryptographie pour construire des algorithmes de chiffrement par bloc. Un critère important sur ces fonctions est une résistance élevée à la cryptanalyse différentielle. Nyberg [20] a défini la notion de la non-linéarité presque parfaite (APN) qui caractérise les fonctions qui ont la meilleure résistance aux attaques différentielles.

Une fonction booléenne $f$ à $m$ variables est d'autant plus résistante aux attaques différentielles que la valeur de $\delta$ est plus petite où

$$
\delta=\sup _{\alpha \neq 0, \beta} \#\left\{x \in \mathbb{F}_{2}^{m} \mid f(x+\alpha)+f(x)=\beta\right\} .
$$

Les fonctions APN sont celles qui atteignent la plus petite valeur de $\delta$, c'està-dire 2.

Jusqu'ici, l'étude des fonctions APN a surtout été consacrée aux fonctions puissances (voir par exemple [6, 11, 12, 13]). Récemment l'étude s'est élargie à d'autres fonctions, en particulier aux polynômes quadratiques (Pott, Carlet et al. [14, 2]) ou à des polynômes sur des petits corps (Dillon [10]).

Divers auteurs (Berger, Canteaut, Charpin, Laigle-Chapuy [1], Byrne et McGuire 3, Jedlicka [16] ou Voloch [22]) ont démontré l'impossibilité de la propriété pour une fonction d'être APN dans certains cas.

Nous montrons ici que pour beaucoup de fonctions polynomiales APN $f$ sur $\mathbb{F}_{2^{m}}$, le nombre $m$ est borné par une expression dépendant du degré de $f$.

Nous utilisons pour cela une méthode déjà initiée par H. Janwa qui a montré, en utilisant la borne de Weil, que certains codes cycliques ne pouvaient pas corriger deux erreurs [18]. A. Canteaut a montré en utilisant la même méthode que certaines fonctions puissances n'étaient pas APN pour une valeur trop grande de l'exposant [5]. Nous avons pu généraliser ce résultat à tous les polynômes en utilisant des résultats de Lang-Weil et de P. Deligne (ou plus exactement des améliorations, due à Ghorpade et Lachaud) sur les conjectures de Weil.

Nous terminons cette article par l'étude des polynômes de petit degré.

Une partie de ces résultats a fait l'objet d'une publication partielle pour le colloque BFCA08. 


\section{Préliminaires}

Pour $a \in \mathbb{F}_{2}^{m *}$, l'équation $f(x+a)+f(x)=b$ a évidemment un nombre pair de solutions, et en a au moins une pour un $b$ dans $\mathbb{F}_{2}^{m}$. D'où la définition suivante.

Définition 2.1 Une fonction $f: \mathbb{F}_{2}^{m} \longrightarrow \mathbb{F}_{2}^{m}$ est $A P N$ si et seulement si pour tout $a \in \mathbb{F}_{2}^{m *}$ et tout $b \in \mathbb{F}_{2}^{m}$, l'équation

$$
f(x+a)+f(x)=b
$$

n'a au plus que 2 solutions.

\subsection{Polynômes équivalents}

Posons $q=2^{m}$. Au lieu de fonctions dans $\mathbb{F}_{2}^{m}$, on parlera de polynômes dans $\mathbb{F}_{q}$, ce qui est équivalent pour les polynômes de degré au plus $2^{m}-1$.

Un polynôme $q$-affine est un polynôme dont les monômes sont de degré 0 ou une puissance de 2 . Les propositions suivantes sont claires.

Proposition 2.1 La classe des fonctions APN est invariante par addition d'un polynôme q-affine.

Nous prendrons désormais pour $f$ une application polynomiale de $\mathbb{F}_{2^{m}}$ dans lui même qui n'ait pas de termes de degré une puissance de 2 ni de terme constant.

Proposition 2.2 Pour tout $a, b$ et $c$ dans $\mathbb{F}_{q}$ le polynôme $c f(a x+b)$ est $A P N$ si et seulement si le polynôme f l'est.

\subsection{L'équivalence au sens de Carlet-Charpin-Zinoviev}

Carlet, Charpin et Zinoviev ont définit une relation d'équivalence entre fonctions booléennes [8]. Pour une fonction $f$ de $\mathbb{F}_{2}^{m}$ dans lui-même on note $G_{f}$ le graphe de la fonction $f$ :

$$
G_{f}=\left\{(x, f(x)) \mid x \in \mathbb{F}_{2}^{m}\right\} .
$$

Définition 2.2 On dit que les fonctions $f, f^{\prime}: \mathbb{F}_{2}^{m} \longrightarrow \mathbb{F}_{2}^{m}$ sont équivalentes au sens de Carlet-Charpin-Zinoviev (on parle aussi de CCZ équivalence) s'il existe une permutation linéaire $L: \mathbb{F}_{2}^{2 m} \longrightarrow \mathbb{F}_{2}^{2 m}$ telle que $L\left(G_{f}\right)=G_{f^{\prime}}$.

D'après [8] si $f$ et $f^{\prime}$ sont CCZ équivalentes, alors $f$ est APN si et seulement si $f^{\prime}$ l'est. 


\subsection{Les monômes APN connus}

Il sont décrits dans la monographie de Carlet [7] où on trouvera les références des démonstrations.

Les fonctions $f(x)=x^{d}$ suivantes sont APN sur $\mathbb{F}_{2^{m}}$, où $d$ est donné par :

$-d=2^{h}+1$ où $\operatorname{pgcd}(h, m)=1$ (fonctions de Gold).

$-d=2^{2 h}-2^{h}+1$ où $\operatorname{pgcd}(h, m)=1$ (fonctions de Kasami).

$-d=2^{(m-1) / 2}+3$ avec $m$ impair (fonctions de Welch).

$-d=2^{(m-1) / 2}+2^{(m-1) / 4}-1$, où $m \equiv 1(\bmod .4)$,

$d=2^{(m-1) / 2}+2^{(3 m-1) / 4}-1$, où $m \equiv 3(\bmod .4)$ (fonctions de Niho)

$-d=2^{m}-2$, pour $m$ impair

$-d=2^{4 m / 5}+2^{3 m / 5}+2^{2 m / 5}+2^{m / 5}-1$, où $m$ est divisible par 5 (fonctions de Dobbertin).

Les fonctions de Gold et de Kasami sont les seules connues où $d$ est indépendant de $m$ et qui donnent des fonctions APN pour une infinité de valeurs de $m$.

\section{Caractérisation des fonctions APN}

Proposition 3.1 La fonction $f: \mathbb{F}_{2^{m}} \longrightarrow \mathbb{F}_{2^{m}}$ est $A P N$ si et seulement si la surface affine

$$
f\left(x_{0}\right)+f\left(x_{1}\right)+f\left(x_{2}\right)+f\left(x_{0}+x_{1}+x_{2}\right)=0
$$

a tous ses points rationnels contenus dans la surface $\left(x_{0}+x_{1}\right)\left(x_{2}+x_{1}\right)\left(x_{0}+\right.$ $\left.x_{2}\right)=0$.

Démonstration. -

Pour que la fonction $f: \mathbb{F}_{2^{m}} \longrightarrow \mathbb{F}_{2^{m}}$ soit APN il faut et il suffit que pour tout $\alpha \in \mathbb{F}_{2}^{m}, \alpha \neq 0$ et pour tout $\beta \in \mathbb{F}_{2}^{m}$,

$$
\#\left\{x_{0} \in \mathbb{F}_{2}^{m}: f\left(x_{0}\right)+f\left(x_{1}\right)=\beta, x_{0}+x_{1}=\alpha\right\} \leq 2
$$

c'est-à-dire que pour tout $\alpha \in \mathbb{F}_{2}^{m}, \alpha \neq 0$ et pour tout $\beta \in \mathbb{F}_{2}^{m}$, il n' y ait pas 4 éléments distincts $x_{0}, x_{1}, x_{2}, x_{3}$ de $\mathbb{F}_{2}^{m}$ qui vérifient

$$
\left\{\begin{array}{l}
x_{0}+x_{1}=\alpha, f\left(x_{0}\right)+f\left(x_{1}\right)=\beta \\
x_{2}+x_{3}=\alpha, f\left(x_{2}\right)+f\left(x_{3}\right)=\beta
\end{array}\right.
$$


Cela revient à dire qu'il n'y a pas 3 éléments distincts $x_{0}, x_{1}, x_{2}$ de $\mathbb{F}_{2}^{m}$ qui vérifient

$$
f\left(x_{0}\right)+f\left(x_{1}\right)+f\left(x_{2}\right)+f\left(x_{0}+x_{1}+x_{2}\right)=0
$$

autrement dit que la surface affine

$$
f\left(x_{0}\right)+f\left(x_{1}\right)+f\left(x_{2}\right)+f\left(x_{0}+x_{1}+x_{2}\right)=0
$$

a tous ses points rationnels contenus dans la surface $\left(x_{0}+x_{1}\right)\left(x_{2}+x_{1}\right)\left(x_{0}+\right.$ $\left.x_{2}\right)=0$.

Avant d'énoncer un corollaire, remarquons que le polynôme $f\left(x_{0}\right)+f\left(x_{1}\right)+$ $f\left(x_{2}\right)+f\left(x_{0}+x_{1}+x_{2}\right)$ est divisible par $\left(x_{0}+x_{1}\right)\left(x_{2}+x_{1}\right)\left(x_{0}+x_{2}\right)$, par conséquent le quotient

$$
\frac{f\left(x_{0}\right)+f\left(x_{1}\right)+f\left(x_{2}\right)+f\left(x_{0}+x_{1}+x_{2}\right)}{\left(x_{0}+x_{1}\right)\left(x_{2}+x_{1}\right)\left(x_{0}+x_{2}\right)}
$$

définit bien un polynôme de degré $d-3$ où $d$ est le degré de $f$. On vérifie facilement que ce polynôme est nul si et seulement si $f$ est un polynôme $q$-affine.

Corollaire 3.1 Si l'application polynomiale $f$ (de degré $d \geq 5$ ) est APN et si la surface affine $X$

$$
\frac{f\left(x_{0}\right)+f\left(x_{1}\right)+f\left(x_{2}\right)+f\left(x_{0}+x_{1}+x_{2}\right)}{\left(x_{0}+x_{1}\right)\left(x_{2}+x_{1}\right)\left(x_{0}+x_{2}\right)}=0
$$

est absolument irréductible, alors la surface projective correspondante $\bar{X}$ admet au plus $4((d-3) q+1)$ point rationnels, où d est le degré de $f$ et $q=2^{m}$.

Démonstration. -

Si $f$ est APN, alors $f$ n'est pas un polynôme q-affine et l'équation cidessus définit bien une surface de degré plus grand que 2. Si la surface $\bar{X}$ contenait le plan $x_{0}+x_{1}=0$, elle serait égale à ce plan puisque la surface $\bar{X}$ est irréductible et serait de degré 1 , ce qui est contraire à l'hypothèse. Par conséquent, elle coupe le plan $x_{0}+x_{1}=0$ suivant une courbe de degré $d-3$. Cette courbe admet au plus $(d-3) q+1$ points rationnels d'après Serre [21]. De même pour le plan à l'infini.

Si $f$ est APN, la surface $\bar{X}$ n'a pas d'autre points rationnels que ceux de la surface $\left(x_{0}+x_{1}\right)\left(x_{2}+x_{1}\right)\left(x_{0}+x_{2}\right)=0$, qui est réunion du plan $x_{0}+x_{1}=0$ et de ses symétriques, ou du plan à l'infini. Donc elle admet au plus $4((d-3) q+1)$ point rationnels. 


\section{Borne inférieure pour le degré d'un poly- nôme APN}

\subsection{Une première borne}

Théorème 4.1 Soit $f$ une application polynomiale de $\mathbb{F}_{2^{m}}$ dans lui même, $d$ son degré. Supposons que la surface $X$ d'équation affine

$$
\frac{f\left(x_{0}\right)+f\left(x_{1}\right)+f\left(x_{2}\right)+f\left(x_{0}+x_{1}+x_{2}\right)}{\left(x_{0}+x_{1}\right)\left(x_{2}+x_{1}\right)\left(x_{0}+x_{2}\right)}=0
$$

soit absolument irréductible. Alors, si $d<0,45 q^{1 / 4}+0,5$ et $d \geq 9$, f n'est pas APN.

Démonstration. -

D'une amélioration d'un résultat de Lang-Weil [19] par Ghorpade-Lachaud ([15, section 11]), on déduit

$$
\left|\bar{X}\left(\mathbb{F}_{2^{m}}\right)-q^{2}-q-1\right| \leq(d-4)(d-5) q^{3 / 2}+18 d^{4} q .
$$

D'où

$$
\bar{X}\left(\mathbb{F}_{2^{m}}\right) \geq q^{2}+q+1-(d-4)(d-5) q^{3 / 2}-18 d^{4} q
$$

Par conséquent, si $q^{2}+q+1-(d-4)(d-5) q^{3 / 2}-18 d^{4} q>4((d-3) q+1)$, alors $\bar{X}\left(\mathbb{F}_{2^{m}}\right)>4((d-3) q+1)$, et donc $f$ n'est pas APN.

Cette condition s'écrit

$$
q-(d-4)(d-5) q^{1 / 2}+\left(-18 d^{4}-4 d+13\right)-3 / q>0
$$

La condition est vérifiée pour $q^{1 / 2}>13,51-5 d+4,773 d^{2}$ si $d \geq 2$. Ou encore pour $d<0,45 q^{1 / 4}+0,5$ et $d \geq 9$.

\subsubsection{Irréductibilité de $X$}

La proposition suivante donne un critère pour que la surface $X$ soit irréductible.

Proposition 4.1 Soit $f$ une application polynomiale de $\mathbb{F}_{2^{m}}$ dans lui même, $d$ son degré. Supposons que la courbe $X_{\infty}$ d'équation

$$
\frac{x_{0}^{d}+x_{1}^{d}+x_{2}^{d}+\left(x_{0}+x_{1}+x_{2}\right)^{d}}{\left(x_{0}+x_{1}\right)\left(x_{2}+x_{1}\right)\left(x_{0}+x_{2}\right)}=0
$$


soit absolument irréductible. Alors la surface $X$ d'équation (1) est absolument irréductible.

Démonstration. -

L'intersection $X_{\infty}$ de la surface $\bar{X}$ avec le plan à l'infini a comme équation

$$
\frac{x_{0}^{d}+x_{1}^{d}+x_{2}^{d}+\left(x_{0}+x_{1}+x_{2}\right)^{d}}{\left(x_{0}+x_{1}\right)\left(x_{2}+x_{1}\right)\left(x_{0}+x_{2}\right)}=0
$$

puisque $x^{d}$ est la composante de degré $d$ du polynôme $f(x)$. Puisque la courbe $X_{\infty}$ est absolument irréductible il en va de même de la surface $\bar{X}$ donc de $X$.

Janwa, McGuire et Wilson [17] ont étudié la courbe $X_{\infty}$ et ont déduit un certain nombre de cas où elle est absolument irréductible.

Proposition 4.2 La courbe $X_{\infty}$ est absolument irréductible pour les valeurs de $d \equiv 3(\bmod .4)$ et pour les valeurs de $d \equiv 5(\bmod .8)$ et $d>13$.

Remarque 4.1 La proposition 4.1] donne une condition suffisante mais pas nécessaire. Voir la section 5.

On peut aussi regarder l'intersection de $X$ avec le plan $x_{2}=a$ ou avec le plan $x_{1}+x_{2}=a$.

Proposition 4.3 Soit $f$ une application polynomiale de $\mathbb{F}_{2^{m}}$ dans lui même, $d$ son degré. Supposons que la courbe $X_{a}$ d'équation

$$
\frac{f\left(x_{0}\right)+f\left(x_{1}\right)+f(a)+f\left(x_{0}+x_{1}+a\right)}{\left(x_{0}+x_{1}\right)\left(a+x_{1}\right)\left(x_{0}+a\right)}=0
$$

soit absolument irréductible. Alors la surface X d'équation (1) est absolument irréductible.

Proposition 4.4 Soit $f$ une application polynomiale de $\mathbb{F}_{2^{m}}$ dans lui même, d son degré. Supposons que la courbe $Y_{a}$ d'équation

$$
F_{a}(x, y)=\frac{f\left(x_{0}\right)+f\left(x_{1}\right)+f\left(x_{1}+a\right)+f\left(x_{0}+a\right)}{\left(x_{0}+x_{1}\right)\left(x_{0}+x_{1}+a\right)}=0
$$

soit absolument irréductible. Alors la surface $X$ d'équation (1) est absolument irréductible.

C'est la même courbe que Voloch considère dans [22]. 


\subsection{Une deuxième borne}

Sons certaines conditions, on peut obtenir une borne meilleure pour la dimension.

Théorème 4.2 Soit $f$ une application polynomiale de $\mathbb{F}_{2^{m}}$ dans lui même, $d$ son degré. Supposons que la surface projective $\bar{X}$ associée à la surface $X$ d'équation affine

$$
\frac{f\left(x_{0}\right)+f\left(x_{1}\right)+f\left(x_{2}\right)+f\left(x_{0}+x_{1}+x_{2}\right)}{\left(x_{0}+x_{1}\right)\left(x_{2}+x_{1}\right)\left(x_{0}+x_{2}\right)}=0
$$

n'ait que des points singuliers isolés. Alors, si $d \geq 10$ et $d<q^{1 / 4}+4$, f n'est pas APN.

Démonstration. -

Notons $b_{i}$ le $i^{\text {ème }}$ nombre de Betti de $\bar{X}$, c'est-à-dire la dimension de l'espace de cohomologie $\ell$-adique de $\bar{X}$ à support compact, et $b_{i}^{\prime}$ le $i^{\text {ème }}$ nombre de Betti primitif, égal à $b_{i}$ pour $i$ impair et à $b_{i}-1$ pour $i$ pair.

D'après une amélioration d'un résultat de Deligne [9] par GhorpadeLachaud ([15], corollaire 7.2), on en déduit

$$
\begin{aligned}
\left|X\left(\mathbb{F}_{2^{m}}\right)-q^{2}-q-1\right| & \leq b_{1}^{\prime}(2, d-3) q^{3 / 2}+\left(b_{2}(3, d-3)+1\right) q \\
& \leq(d-4)(d-5) q^{3 / 2}+\left(d^{3}-13 d^{2}+57 d-82\right) q
\end{aligned}
$$

D'où

$$
X\left(\mathbb{F}_{2^{m}}\right) \geq q^{2}+q+1-(d-4)(d-5) q^{3 / 2}-\left(d^{3}-13 d^{2}+57 d-82\right) q .
$$

Par conséquent, si

$q^{2}+q+1-(d-4)(d-5) q^{3 / 2}-\left(d^{3}-13 d^{2}+57 d-82\right) q>3((d-3) q+1)$

alors $X\left(\mathbb{F}_{2^{m}}\right)>4((d-3) q+1)$, et donc $f$ n'est pas APN. Cette condition s'écrit

$$
q+\left(-d^{2}+9 d-20\right) q^{1 / 2}+\left(-d^{3}+13 d^{2}-61 d+95\right)-\frac{2}{q}>0
$$

La condition est vérifiée pour $q>d^{4}-16 d^{3}+94 d^{2}-228 d+173$ dès que $d \geq 6$. Ou encore pour $d<q^{1 / 4}+4$ dès que $d \geq 10$. 


\subsubsection{Non-singularité de $X_{\infty}$}

Théorème 4.3 Soit $f$ une application polynomiale de $\mathbb{F}_{2^{m}}$ dans lui même, $d$ son degré. Supposons que la courbe $X_{\infty}$ d'équation

$$
\frac{x_{0}^{d}+x_{1}^{d}+x_{2}^{d}+\left(x_{0}+x_{1}+x_{2}\right)^{d}}{\left(x_{0}+x_{1}\right)\left(x_{2}+x_{1}\right)\left(x_{0}+x_{2}\right)}=0
$$

soit lisse. Alors la surface $\bar{X}$ n'a que des points singuliers isolés.

Demonstration

La démonstration du théorème 4.1 montre que $X_{\infty}$ est l'intersection de $X$ avec l'hyperplan à l'infini. Puisque la courbe $X_{\infty}$ est absolument irréductible il en va de même de la surface $\bar{X}$.

On peut déduire que la surface $\bar{X}$ est régulière en codimension 1 (c'està-dire n'a que des points singuliers isolés) si la courbe $X_{\infty}$ est non-singulière (cf. Ghorpade-Lachaud [15], corollaire 1.4).

Janwa, et Wilson [18] ont étudié la courbe $X_{\infty}$ et ont déduit un certain nombre de cas où elle est non singulière.

Proposition 4.5 La courbe $X_{\infty}$ est non singulière pour les valeurs de $d=$ $2 l+1$ où

- $l$ est un entier impair tel qu'il existe un entier $r$ avec $2^{r} \equiv-1$ (mod. $l$ ).

- l est un nombre premier plus grand que 17 tel que l'ordre de 2 modulo $l$ soit $(l-1) / 2$.

En particulier la première condition est satisfaite si $l$ est un nombre premier congru à \pm 3 modulo 8 . Parmi les degrés inférieurs à 100, on trouve $7,11,19$, $23,27,35,39,47,51,55,59,67,75,83,95$.

\subsection{La surface $X$ ne peut pas être lisse}

On pourrait se poser la question de savoir si la surface $\bar{X}$ peut être lisse, ce qui améliorerait encore les bornes sur le degré des fonctions APN. Ce ne peut être le cas. Soit en effet

$$
\phi\left(x_{0}, x_{1}, x_{2}\right)=\frac{f\left(x_{0}\right)+f\left(x_{1}\right)+f\left(x_{2}\right)+f\left(x_{0}+x_{1}+x_{2}\right)}{\left(x_{0}+x_{1}\right)\left(x_{2}+x_{1}\right)\left(x_{0}+x_{2}\right)}
$$

l'équation affine de la surface $X$. Les points singuliers de cette surface $X$ sont sur les surfaces d'équation $\phi_{x_{i}}^{\prime}\left(x_{0}, x_{1}, x_{2}\right)=0$ où on note $\phi_{x_{i}}^{\prime}$ la dérivée de $\phi$ par rapport à $x_{i}$. 
Lemme 4.1 Le polynôme $x_{1}+x_{2}$ divise $\phi_{x_{0}}^{\prime}\left(x_{0}, x_{1}, x_{2}\right)=0$.

\section{Démonstration}

Il suffit de montrer le lemme pour chaque monôme de $f$. Si on fait le changement de variable $x_{1}+x_{2}=t$, le polynôme $\phi$ devient, pour un monôme de degré $r$

$$
\begin{aligned}
\psi\left(x_{0}, x_{1}, t\right) & =\frac{x_{0}^{r}+x_{1}^{r}+\left(x_{1}+t\right)^{r}+\left(x_{0}+t\right)^{r}}{\left(x_{0}+x_{1}\right)\left(x_{0}+x_{1}+t\right) t} \\
& =\frac{r\left(x_{1}^{r-1}+x_{0}^{r-1}\right)+t g\left(x_{0}, x_{1}, t\right)}{\left(x_{0}+x_{1}\right)\left(x_{0}+x_{1}+t\right)}
\end{aligned}
$$

où $g\left(x_{0}, x_{1}, t\right)$ est un polynôme. En dérivant par rapport à $x_{0}$, cela donne

$$
\psi_{x_{0}}^{\prime}\left(x_{0}, x_{1}, t\right)=\frac{r(r-1)\left(x_{0}^{r}+x_{0}^{r-2} x_{1}^{2}\right)+t g_{1}\left(x_{0}, x_{1}, t\right)}{\left(x_{0}+x_{1}\right)^{2}\left(t+x_{0}+x_{1}\right)^{2}}
$$

où $g_{1}\left(x_{0}, x_{1}, t\right)$ est un polynôme. Cela montre le lemme car $r(r-1) \equiv$ 0 (mod. 2).

Par conséquent l'intersection de la droite $x_{0}=x_{1}=x_{2}$ avec la surface affine $X$ est formé de points singuliers de $X$.

Si la surface projective $\bar{X}$ ne rencontre pas la droite en des points à distance finie, elle ont un point commun d'ordre $d-3$ à l'infini. L'équation $\phi(u, u, u)=0$ n'a pas de solution, donc $\phi(u, u, u)$ est une constante non-nulle $a_{0}$. Soit $\Phi\left(x_{0}: x_{1}: x_{2}: z\right)$ l'équation projective de la surface $\bar{X}$. Elle est égale à $z^{d-3} \phi\left(\frac{x_{0}}{z}, \frac{x_{1}}{z}, \frac{x_{2}}{z}\right)$, donc on a $\Phi(1,1,1, z)=z^{d-3} \phi\left(\frac{1}{z}, \frac{1}{z}, \frac{1}{z}\right)=z^{d-3} a_{0}$. La formule

$$
x_{0} \Phi_{x_{0}}^{\prime}+x_{1} \Phi_{x_{1}}^{\prime}+x_{2} \Phi_{x_{2}}^{\prime}+z \Phi_{z}^{\prime}=(d-3) \Phi\left(x_{0}, x_{1}, x_{2}, z\right)
$$

restreinte à la droite $x_{0}=x_{1}=x_{2}$ devient donc $z \Phi_{z}^{\prime}=(d-3) z^{d-3} a_{0}$, d'où $\Phi_{z}^{\prime}=(d-3) z^{d-4} a_{0}$ sur cette droite. Par conséquent $\Phi_{z}^{\prime}(1,1,1,0)=0$ et le point $(1,1,1,0)$ est bien singulier si $d \geq 5$. 


\section{Autres exemples}

\subsection{Binômes}

Proposition 5.1 Soient $d$ et $r$ deux entiers tels que $d>r \geq 3$, et soit $f(x)=x^{d}+a x^{r}$, avec $a \in \mathbb{F}_{q}$. Soit $\phi_{s}$ le polynôme

$$
\frac{x_{0}^{s}+x_{1}^{s}+x_{2}^{s}+\left(x_{0}+x_{1}+x_{2}\right)^{s}}{\left(x_{0}+x_{1}\right)\left(x_{2}+x_{1}\right)\left(x_{0}+x_{2}\right)}
$$

pour $s=d$ ou $r$. Supposons que $\left(\phi_{d}, \phi_{r}\right)=1$ et que

- ou bien $\phi_{d}$ se décompose en facteurs distincts sur $\overline{\mathbb{F}}_{2^{m}}$ et $r \geq 5$;

- ou bien $\phi_{r}$ se décompose en facteurs distincts sur $\overline{\mathbb{F}}_{2^{m}}$.

Alors, si $d<0,45 q^{1 / 4}+0,5$ et $d \geq 9$, f n'est pas $A P N$.

\section{Démonstration}

L'équation de la surface $X$ associée à $f$ est $\phi_{d}+a \phi_{r}=0$. Le lemme suivant montre que cette surface est irréductible sous les hypothèses faites.

Lemme 5.1 Soit $\Phi(x, y, z) \in \mathbb{F}_{2^{m}}[x, y, z]$ la somme de deux polynômes homogènes, c'est-à-dire $\Phi=\Phi_{r}+\Phi_{d}$ où $\Phi_{i}$ est homogène de degré $i$, et $r<d$. Supposons que $\left(\Phi_{r}, \Phi_{d}\right)=1$ et que

- ou bien $\Phi_{r}$ se décompose en facteurs distincts sur $\overline{\mathbb{F}}_{2^{m}}$ et $r \geq 1$;

- ou bien $\Phi_{d}$ se décompose en facteurs distincts sur $\overline{\mathbb{F}}_{2^{m}}$ et $r \geq 0$. Alors $\Phi$ est absolument irréductible sur $\mathbb{F}_{2^{m}}$.

\section{Démonstration -}

On fait le même raisonnement que Byrne et McGuire dans l'article [3. lemme 2]. La preuve fonctionne aussi dans le cas des polynômes à trois variables. Elle fonctionne aussi dans le deuxième cas du lemme $\left(\Phi_{d}\right.$ se décompose en facteurs distincts sur $\overline{\mathbb{F}}_{2^{m}}$ et $r \geq 0$ ).

Par exemple, la proposition 5.1 montre que le polynôme $x^{13}+a x^{7}$ avec $a \neq 0$ ne peut être APN que si $m \leq 19$, car le polynôme $\phi_{7}$ est irréductible et ne divise pas $\phi_{13}$ d'après [18]. 
On a aussi la proposition suivante due pour l'essentiel à F. Voloch.

Proposition 5.2 Soit $f(x)=x^{d}+c x^{r}$, où $c \in \overline{\mathbb{F}}_{2}^{*}, r<d$ sont des entiers, non tous les deux pairs, et non plus une puissance de 2 et tels que $(d-1, r-1)$ soit une puissance de 2. Alors, si $d<0,45 q^{1 / 4}+0,5$ et $d \geq 9$, $f$ n'est pas $A P N$.

Démonstration -

F. Voloch a montré dans [22] que ces hypothèses impliquaient que la courbe

$$
F_{a}(x, y)=\frac{f\left(x_{0}\right)+f\left(x_{1}\right)+f\left(x_{1}+a\right)+f\left(x_{0}+a\right)}{\left(x_{0}+x_{1}\right)\left(x_{0}+x_{1}+a\right)}
$$

est irréductible dans $\mathbb{F}_{2}[x, y, a]$.

Remarque 5.1 L'énoncé de cette proposition [22, Theorem 3] supposait que $r$ et d étaient premiers entre eux. F. Voloch m'a communiqué que ce n'était pas nécessaire.

\subsection{Polynômes de degré 3 ou 5}

D'après la proposition 2.1, il suffit de regarder les polynômes de la forme $a_{5} x^{5}+a_{3} x^{3}$. Ces polynômes sont combinaison linéaires de plusieurs monômes de la forme $x^{2^{i}+1}$ et ils ne peuvent pas être APN d'après [1] sauf si $a_{3}$ ou $a_{5}$ est nul, auquel cas ce sont des fonctions de Gold.

\subsection{Polynômes de degré 6}

Proposition 5.3 Soit $f(x)=x^{6}+a_{5} x^{5}+a_{3} x^{3}$ un polynôme de degré 6 . Le polynôme $f$ est APN si et seulement si $a_{3}=a_{5}=0$. Il est alors équivalent à un fonction de Gold.

Démonstration -

Il est facile de vérifier que la surface $X$ associée ne contient un hyperplan que si $a_{3}=a_{5}^{3}$. Donc elle est absolument irréductible, sauf si $a_{3}=a_{5}^{3}$. Dans ce cas-ci, on vérifie que la surface $X$ se décompose en 3 hyperplans d'équation $x_{0}+x_{2}+a_{5}=0, x_{0}+x_{1}+a_{5}=0, a_{5}+x_{1}+x_{2}=0$ et la fonction $f$ n'est pas APN si $a_{5} \neq 0$, d'après la proposition 3.1 . 
Si $a_{3} \neq a_{5}^{3}$ et $a_{3}=0$, on a donc $f(x)=x^{6}+a_{5} x^{5}$. Comme $a_{5} \neq 0$, on se ramène par une transformation affine (cf. proposition 2.2) à $a_{5}^{-6} f\left(a_{5} x\right)=$ $x^{6}+x^{5}$. Des points de la surface affine $X$ sont alors

$$
\left(\frac{1}{\lambda(1+\lambda)}, \frac{\lambda^{3}}{\lambda(1+\lambda)}, 1\right)
$$

avec $\lambda \in \mathbb{F}_{q}-\mathbb{F}_{2}$. Si $m \geq 3$, ils ne sont pas sur la surface d'équation $\left(x_{0}+\right.$ $\left.x_{1}\right)\left(x_{2}+x_{1}\right)\left(x_{0}+x_{2}\right)=0$, donc la fonction $f(x)=x^{6}+a_{5} x^{5}$ avec $a_{5} \neq 0$ n'est jamais APN, d'après la proposition 3.1 .

Si $a_{3} \neq a_{5}^{3}$ et si, de plus, $a_{3} \neq 0$, alors la surface $\bar{X}$ n'a que des singularités isolées et la fonction $f$ ne peut être APN que si $m \leq 4$ d'après la démonstration du théorème 4.2. D'après [4, ces fonctions ne peuvent pas être APN.

\subsection{Polynômes de degré 7}

Proposition 5.4 Soit $f$ un polynôme de degré 7. Pour $m \geq 3$, le polynôme $f$ ne peut être $A P N$ que s'il est CCZ-équivalent au polynôme $x^{7}$ sur $\mathbb{F}_{32}$. Il est alors équivalent à un fonction de Welsh.

\section{Démonstration -}

Le théorème 4.3 et la proposition 4.5 montrent que la surface $\bar{X}$ n'a que des points singuliers isolés. D'après la démonstration du théorème 4.2 on en déduit que $f$ ne peut être APN que si $m \leq 6$. La proposition s'ensuit, d'après [4] et avec une recherche exhaustive pour $m=6$.

\subsection{Polynômes de degré 9}

Proposition 5.5 Soit $f$ un polynôme de degré 9. Le polynôme $f$ ne peut être $A P N$ pour une infinité de $m$ que s'il est équivalent au polynôme $x^{9}$. Il est alors $C C Z$-équivalent à un fonction de Gold. Autrement le polynôme $f$ ne peut être $A P N$ que pour $m=6$ et il est égal à une fonction $f=x^{9}+a_{6} x^{6}+a_{3} x^{3}$ ou à une fonction CCZ-équivalente.

\section{Démonstration -}

On peut se limiter grce aux proposition 2.1 et 2.2 aux polynômes

$$
f(x)=x^{9}+a_{7} x^{7}+a_{6} x^{6}+a_{5} x^{5}+a_{3} x^{3} .
$$


Si $a_{7}=0$, on obtient

$$
f(x)=x^{9}+a_{6} x^{6}+a_{5} x^{5}+a_{3} x^{3} .
$$

Si, de plus, $a_{6}=0$, on obtient un polynôme $f(x)=x^{9}+a_{5} x^{5}+a_{3} x^{3}$ dont tous les degrés des monômes sont de la forme $2^{r}+1$. D'après [1] il ne peut par être APN, sauf si c'est un monôme. Si $a_{6} \neq 0$, on peut réduire l'étude, grce à la proposition [2.2, aux polynômes $f(x)=x^{9}+a_{6} x^{6}+x^{5}+a_{3} x^{3}$ et $f=x^{9}+a_{6} x^{6}+a_{3} x^{3}$.

Si $a_{7} \neq 0$ on obtient, d'après les mêmes propositions le polynôme

$$
f(x)=x^{9}+x^{7}+a_{5} x^{5}+a_{3} x^{3} .
$$

Pour $f(x)=x^{9}+x^{7}+a_{5} x^{5}+a_{3} x^{3}$, on vérifie aisément que la surface $\bar{X}$ n'a que des singularités isolées (voir ci-dessous). La fonction $f$ ne peut être APN que si $m \leq 13$, d'après la démonstration du théorème 4.2. La fonction $x^{9}$ est un fonction de Gold. Pour $f(x)=x^{9}+a_{6} x^{6}+a_{3} x^{3}$ avec $a_{3}=a_{6}^{2} \neq 0$ la fonction $f$ ne peut être APN ; pour $f(x)=x^{9}+a_{6} x^{6}+a_{5} x^{5}+a_{3} x^{3}$ avec $a_{3}$, $a_{5}$ ou $a_{6}$ non nuls, la fonction $f$ ne peut être APN que si $m \leq 8$ (voir 5.5.2). Un examen exhaustif des cas restant montre la proposition. La seule fonction APN supplémentaire que l'on trouve est une fonction $f=x^{9}+a_{6} x^{6}+a_{3} x^{3}$ pour $m=6$ déjà obtenue par Dillon [10].

\subsubsection{Points singuliers de $X$ associé à $x^{9}+x^{7}+a_{5} x^{5}+a_{3} x^{3}$.}

La surface $\bar{X}$ a pour équation projective $B_{6}+B_{4} z^{2}+a_{5} B_{2} z^{4}+a_{3} z^{6}$ où les $B_{i}$ sont des polynômes homogènes de degré $i$ en $x_{0}, x_{1}, x_{2}$ et ce sont les équations des courbes $X_{\infty}$ correspondantes aux degrés $i$. Par [18], les polynômes $B_{6}$ et $B_{2}$ sont des équations de réunions de droites passant par le point $(1: 1: 1)$, définies sur $\mathbb{F}_{8}$ et $\mathbb{F}_{4}$ et le polynôme $B_{4}$ est l'équation d'une courbe irréductible. D'après le calcul des dérivées de $X$, on étudie plusieurs cas.

Cas $\mathrm{n}^{\circ} 1: x_{0}=x_{1}=x_{2}$. Les points de la surface $X$ qui sont sur cette droite sont ceux qui vérifient $x_{0}^{4}+a_{3} z^{4}=0$ ou $z=0$.

Cas $\mathrm{n}^{\circ} 2: x_{1}=x_{2}$. Les points singuliers de la surface $X$ qui sont sur ce plan sont ceux qui vérifient $x_{0}^{4}+x_{2}^{4}+x_{0} x_{2} z^{2}+a_{5} z^{4}=0$ et $x_{0}^{6}+x_{0}^{4} x_{2}^{2}+x_{0}^{2} x_{2}^{4}+$ $x_{2}^{6}+x_{0}^{4} z^{2}+x_{0}^{2} x_{2}^{2} z^{2}+x_{2}^{4} z^{2}+a_{5} x_{0}^{2} z^{4}+a_{5} x_{2}^{2} z^{4}+a_{3} z^{6}=0$ qui est 2 fois la courbe $\left(x_{0}+x_{2}\right)^{3}+\left(x_{0}^{2}+x_{0} x_{2}+x_{2}^{2}\right) z+a_{5}\left(x_{0}+x_{2}\right) z^{2}+a_{3} z^{3}=0$. Un changement de variable $s=x_{0}+x_{2}$ donne, pour $z=1: s^{4}+x_{0}\left(x_{0}+s\right)+a_{5}=0$ 
et $s^{3}+s^{2}+x_{0}\left(s+x_{0}\right)+a_{5} s+a_{3}=0$ qui sont deux courbes elliptiques distinctes. Les deux courbes ne se coupent qu'en un nombre fini de points.

Cas $n^{\circ} 3: x_{0} \neq x_{1} \neq x_{2} \neq x_{0}$.

Les points singuliers de la surface $X$ sont ceux qui vérifient

$$
\left\{\begin{array}{l}
a_{5}+x_{1} x_{2}+B_{2}^{2}\left(x_{0}, x_{1}, x_{2}\right)=0 \\
a_{5}+x_{0} x_{2}+B_{2}^{2}\left(x_{0}, x_{1}, x_{2}\right)=0 \\
a_{5}+x_{1} x_{0}+B_{2}^{2}\left(x_{0}, x_{1}, x_{2}\right)=0
\end{array}\right.
$$

donc, par soustraction, qui vérifient

$$
\left\{\begin{array}{l}
\left(x_{1}+x_{0}\right) x_{2}=0 \\
\left(x_{2}+x_{1}\right) x_{0}=0 \\
\left(x_{0}+x_{2}\right) x_{1}=0
\end{array}\right.
$$

D'après l'hypothèse, on a donc $x_{0}=x_{1}=x_{2}$, donc on n'a rien de mieux.

\subsubsection{Surface associée à $x^{9}+a_{6} x^{6}+a_{5} x_{5}+a_{3} x^{3}$.}

Si $a_{3}=a_{5}=a_{6}=0$, la fonction $x^{9}$ est une fonction de Gold. Supposons que l'un de ces coefficient soit non nul.

La surface $X$ a pour équation projective $B_{6}+a_{6} B_{3} z^{3}+a_{5} B_{2} z^{4}+a_{3} z^{6}$ où les $B_{i}$ sont comme plus haut. L'intersection de la surface $X$ avec le plan $x_{2}=0$ est la courbe $Y$ d'équation affine

$$
\phi\left(x_{0}, x_{1}, 0\right)=B_{6}\left(x_{0}, x_{1}, 0\right)+a_{6} B_{3}\left(x_{0}, x_{1}, 0\right)+a_{5} B_{2}\left(x_{0}, x_{1}, 0\right)+a_{3}
$$

avec $B_{6}\left(x_{0}, x_{1}, 0\right)=\prod_{\beta \in \mathbb{F}_{8}-\mathbb{F}_{4}}\left(x_{0}+\beta x_{1}\right), B_{3}\left(x_{0}, x_{1}, 0\right)=x_{0} x_{1}\left(x_{0}+x_{1}\right)$ et $B_{2}\left(x_{0}, x_{1}, 0\right)=\left(x_{0}+\alpha x_{1}\right)\left(x_{0}+\alpha^{2} x_{1}\right)$ où $\alpha \in \mathbb{F}_{4}-\mathbb{F}_{2}$.

Les raisonnements de Byrne et McGuire pour prouver le lemme 2 dans [3] peuvent s'appliquer. On trouve ainsi que le seul cas où $\phi\left(x_{0}, x_{1}, 0\right)$ pourrait être réductible est :

$$
\phi\left(x_{0}, x_{1}, 0\right)=\left(P_{3}+P_{0}\right)\left(Q_{3}+Q_{0}\right)=P_{3} Q_{3}+P_{3} Q_{0}+P_{0} Q_{3}+P_{0} Q_{0}
$$

où les $P_{i}$ et les $Q_{i}$ soient des polynômes homogènes de degré $i$. De plus le fait que $P_{3} Q_{0}+P_{0} Q_{3}=a_{6} B_{3}\left(x_{0}, x_{1}, 0\right)$ implique que $P_{3}=\left(x_{0}+\beta x_{1}\right)\left(x_{0}+\right.$ $\left.\beta^{2} x_{1}\right)\left(x_{0}+\beta^{4} x_{1}\right), Q_{3}=\left(x_{0}+\beta^{3} x_{1}\right)\left(x_{0}+\beta^{5} x_{1}\right)\left(x_{0}+\beta^{6} x_{1}\right)$, et que $P_{0}=Q_{0}$. Cela implique déjà que si $a_{5} \neq 0$, la courbe $Y$ est indécomposable.

On trouve donc que le seul cas où la courbe $Y$ est réductible est le cas où $a_{3}=a_{6}^{2}$ et $a_{5}=0$. Dans ce cas, la surface $X$ se décompose en $X=X_{1} \cup X_{2}$, 
où $X_{1}$ a pour équation $x_{0}^{3}+x_{0}^{2} x_{1}+x_{1}^{3}+x_{1}^{2} x_{2}+x_{0} x_{2}^{2}+x_{2}^{3}+a_{6}$ et $X_{2}$ a pour équation $x_{0}^{3}+x_{0} x_{1}^{2}+x_{1}^{3}+x_{0}^{2} x_{2}+x_{1} x_{2}^{2}+x_{2}^{3}+a_{6}=0$. L'intersection de la surface $X_{1}$ avec le plan $x_{2}=0$ est une courbe elliptique d'équation affine $1+x_{0}^{3}+x_{0} x_{1}^{2}+x_{1}^{3}=0$. Elle ne peut rencontrer les droites $x_{1}=0$, $x_{2}=0, x_{1}+x_{3}=0$ ou la droite à l'infini qu'en 3 points chacune. Or elle a au moins $1+q-2 \sqrt{q}$ points rationnels d'après la borne de Hasse-Weil, ce qui prouve que la surface affine $X_{1}$ a des points rationnels en dehors de la surface $\left(x_{0}+x_{1}\right)\left(x_{2}+x_{1}\right)\left(x_{0}+x_{2}\right)=0$ pour $1+q-2 \sqrt{q}>12$. Par conséquent, $f(x)=x^{9}+a_{6} x^{6}+a_{6}^{2} x^{3}$ n'est pas APN pour $m \geq 5$. Pour $m \leq 4$, le calcul exhaustif montre que l'on obtient pas de nouvelles fonctions APN (cf. [4]).

Dans le cas où la courbe $Y$ est irréductible, elle a au plus $1+q-20 \sqrt{q}$ points rationnels d'après le corollaire 7.4 de [15]. Si la fonction $f$ est APN, la courbe projective $\bar{Y}$ doit avoir tous ses points rationnels contenus dans la courbe réunion de 4 droites $x_{0} x_{1}\left(x_{0}+x_{1}\right) z=0$. Elle recoupe chaque droite en 6 points au plus, donc elle doit avoir au plus 24 points. Cela n'est possible que si $m \leq 8$.

\section{Applications numériques}

Quand la surface $X$ est irréductible, on obtient que la fonction $f$ ne peut être APN que si $m \leq m_{\max }$ où $m_{\max }$ est donné par le tableau suivant.

\begin{tabular}{|c|c|c|c|c|c|c|c|c|c|c|c|c|c|c|c|}
\hline$d \leq$ & 7 & 9 & 10 & 12 & 15 & 17 & 21 & 23 & 29 & 36 & 41 & 49 & 50 & 70 & 83 \\
\hline$m_{\max }$ & 15 & 16 & 17 & 18 & 19 & 20 & 21 & 22 & 23 & 24 & 25 & 26 & 27 & 28 & 29 \\
\hline
\end{tabular}

Quand la surface $\bar{X}$ est de plus à singularités isolées, on obtient que la fonction $f$ ne peut être APN que si $m \leq m_{\max }$ où $m_{\max }$ est donné par le tableau suivant.

\begin{tabular}{|c|c|c|c|c|c|c|c|c|c|c|c|c|c|c|c|}
\hline$d \leq$ & 7 & 9 & 10 & 12 & 13 & 15 & 17 & 20 & 23 & 26 & 30 & 36 & 42 & 49 & 57 \\
\hline$m_{\max }$ & 6 & 9 & 10 & 11 & 12 & 13 & 14 & 15 & 16 & 17 & 18 & 19 & 20 & 21 & 22 \\
\hline
\end{tabular}

\section{$7 \quad$ Remerciements}

Je remercie Felipe Voloch qui m'a aidé par ses commentaires sur une ancienne version de l'article et Gregor Leander qui m'a aidé pour les recherches exhaustives. 


\section{Références}

[1] T. Berger, A. Canteaut, P. Charpin, Y. Laigle-Chapuy On almost perfect nonlinear functions over $F_{2}^{n}$. IEEE Trans. Inform. Theory 52 (2006), no. 9, 4160-4170.

[2] L. Budaghyan and C. Carlet and P. Felke and G. Leander An infinite class of quadratic APN functions which are not equivalent to power mappings, Cryptology ePrint Archive, $\mathrm{n}^{\circ}$ 2005/359

[3] Byrne E. and McGuire G., On the Non-Existence of Quadratic APN and Crooked Functions on Finite Fields, prepublication. http ://www.maths.may.ie/sta/gmg/APNniceWeilEBGMG.pdf.

[4] Marcus Brinkman, G. Leander : On the classification of APN functions up to dimension five, International Workshop on Coding and Cryptography (WCC), Versailles, France, 2007.

[5] Canteaut, A., Differential cryptanalysis of Feistel ciphers and differentially $\delta$-uniform mappings. In Selected Areas on Cryptography, SAC'97, pages 172-184, Ottawa, Canada, 1997.

[6] A. Canteaut, P. Charpin, and H. Dobbertin. Weight divisibility of cyclic codes, highly nonlinear functions on $G F\left(2^{m}\right)$ and crosscorrelation of maximum-length sequences. SIAM Journal on Discrete Mathematics, 13(1), 2000.

[7] C. Carlet. Vectorial Boolean Functions for Cryptography. Chapter of the monography Boolean Methods and Models, Y. Crama and P. Hammer eds, Cambridge University Press, to appear.

[8] C. Carlet, P. Charpin and V. Zinoviev. Codes, bent functions and permutations suitable for DES-like cryptosystems. Designs, Codes and Cryptography, 15(2), pp. 125-156, 1998.

[9] Deligne, Pierre La conjecture de Weil : I. Publications Mathématiques de l'IHES, 43 (1974), p. 273-307

[10] J. F. Dillon APN Polynomials and Related Codes Conference on Polynomials over Finite Fields and Applications, Banff International Research Station November 2006.

[11] H. Dobbertin. Almost perfect nonlinear power functions over $G F\left(2^{n}\right)$ : the Niho case. Inform. and Comput., 151, pp. 57-72, 1999. 
[12] H. Dobbertin. Almost perfect nonlinear power functions over $G F\left(2^{n}\right)$ : the Welch case. IEEE Trans. Inform. Theory, 45, pp. 1271$1275,1999$.

[13] H. Dobbertin. Almost perfect nonlinear power functions over $G F\left(2^{n}\right)$ : a new case for $n$ divisible by 5 . D. Jungnickel and H. Niederreiter eds. Proceedings of Finite Fields and Applications FQ5, Augsburg, Germany, Springer, pp. 113-121, 2000.

[14] Y. Edel, G. Kyureghyan and A. Pott. A new APN function which is not equivalent to a power mapping. Preprint, 2005, http ://arxiv.org/abs/math.CO/0506420

[15] Ghorpade Sudhir R., Lachaud Gilles. Étale cohomology, Lefschetz theorems and number of points of singular varieties over finite fields., Mosc. Math. J. 2 (2002), n 3, 589-631.

[16] Jedlicka, D., APN monomials over $\mathrm{GF}\left(2^{n}\right)$ for infinitely many $n$, Finite Fields Appl. 13 (2007), no. 4, 1006-1028.

[17] Janwa, H., McGuire, G., Wilson, R., Double-error-correcting cyclic codes and absolutely irreducible polynomials over GF(2). J. Algebra 178 (1995), no. 2, 665-676.

[18] Janwa, H., Wilson, R. M. Hyperplane sections of Fermat varieties in $P^{3}$ in char. 2 and some applications to cyclic codes. Applied algebra, algebraic algorithms and error-correcting codes (San Juan, PR, 1993), 180-194, Lecture Notes in Comput. Sci., 673, Springer, Berlin, 1993.

[19] Lang, Serge, Weil, André Number of points of varieties in finite fields, Amer. J. Math. 76, (1954). 819-827.

[20] Nyberg, Kaisa, Differentially uniform mappings for cryptography. Advances in cryptology -EUROCRYPT '93 (Lofthus, 1993), 55-64, Lecture Notes in Comput. Sci., 765, Springer, Berlin, 1994.

[21] J. -P. Serre, Lettre à M. Tsfasman, Astérisque 198-199-200 (1991), 351-353.

[22] Voloch, F., Symmetric Cryptography and Algebraic Curves, preprint, http ://www.ma.utexas.edu/users/voloch/preprint.html 